\title{
An Introduction to Cognitive Restructuring for COVID-19 Induced Stress in Post-graduate Trainees
}

Saniya Sabzwari ( $\square$ saniya.sabzwari@aku.edu )

Aga Khan University

\section{Rafay lqbal}

Aga Khan University

\section{Research Article}

Keywords: Cognitive restructuring, Cognitive behavior therapy, COVID-19, stress, postgraduate trainees

Posted Date: September 8th, 2021

DOl: https://doi.org/10.21203/rs.3.rs-795127/v1

License: (9) This work is licensed under a Creative Commons Attribution 4.0 International License. Read Full License 


\section{Abstract}

Background: Healthcare professionals have experienced a wide range of psychological problems during the COVID-19 pandemic. Frontline workers and trainees are particularly vulnerable to such effects. This study aimed to share the experience of Cognitive Restructuring workshops for COVID-19 induced Stress in Post-graduate Trainees, highlighting their stress levels and effect of cognitive behavior therapy intervention in processing and managing stress.

Methods: Workshops based on a Cognitive Behavior Therapy model were introduced to trainees at the Aga Khan University Hospital to self-assess stress and identify coping mechanisms. We collected data from the stress scale and workshop responses along with a focus group discussion. We performed item analysis of the stress scale and a documentary analysis of participant responses to assess the effectiveness of this intervention.

Results: The majority of the participants had moderate stress. A statistically significant relationship was observed between stress level and perceived helplessness ( $p$ value 0.002$)$. The feedback of the workshop was largely positive based on sharing of adverse experiences, developing a different perspective and a newer way of processing feelings; about $10 \%$ of the participants did not find this workshop useful. In the focus group discussion, the participants described stress scale to be helpful for stress quantification, ABC tool to be useful with repeated practice and reframing technique to reduce stress over time.

Conclusion: The COVID-19 pandemic has caused stress in healthcare workers and impacted professional and personal lives. Interventions like group cognitive behavior therapy can be useful in recognizing and managing stress in trainees and can be incorporated in training programs to build resilience for similar events.

\section{Background}

The COVID-19 pandemic has caused people to experience a wide range of physical and psychological symptoms (1-3).

Physicians and paramedical staff are the front line of healthcare and therefore at greater risk of exposure to the illness. They are vulnerable to negative mental health effects as they strive to balance the duty of care for patients with concerns about their well-being and that of their families. Nurses and physicians are at greater risk of mental health problems like depression and post-traumatic stress disorder (PTSD) when exposed to catastrophic medical events(4). More than half of healthcare workers reported psychological distress following the severe acute respiratory syndrome (SARS) outbreak(5).

The psychological effects experienced by healthcare professionals in the current pandemic are likely due to the unpredictability of the illness, personal implications to safety and a sudden alteration in the healthcare environment (6). The need for psychological care therefore became increasingly important and support programs have been established (7-9). 
In Pakistan, the Aga Khan University Hospital was the first medical center in the country to activate its emergency response program against COVID-19. The stress of working through this crisis became apparent after several frontline staff got exposed and quarantined. Our postgraduate trainees form a large part of this frontline workforce. To provide support to trainees, stress management workshops were developed and conducted using a cognitive behavior therapy (CBT) approach.

This approach has been used in trainees for self-improvement (10) and is an effective intervention for various kinds of psychological trauma (11). Group CBT has also been shown to be effective in this area (12).

This study shares the trainees' experience of these workshops, highlighting their stress levels and effect of the CBT intervention in processing and managing stress.

\section{Methods}

A mixed method study design was used for this study. All participants completed a 10-item perceived stress scale (PSS)(13). Participants then attended a 90-minute online CBT workshop using the ABC model (Adversity, Beliefs, Consequences) with content derived from the toolkit for emotional coping for healthcare staff (TECHS) developed by the Center for Pediatric Traumatic Stress(14). Six workshops consisting of 10 to 15 participants were conducted from April to August 2020.

All participants completed the stress scale prior to the workshop. The facilitator explained the concept of group CBT and the importance of confidentiality of their shared experiences. The overall stress level of the participants was discussed, and they were guided through the $A B C$ tool followed by steps to reframing and coping.

At the end, the participants submitted workshop feedback based on the Kolb's experiential learning model to assess its effectiveness.

A second workshop was conducted one month later with Family Medicine trainees to see the delayed impact and applicability of $A B C$ model and reframing techniques. This was followed by a focus group discussion (FGD) to get an in-depth perspective of the participants' experience of the workshop. The questions for this were independently develop by the FGD facilitator. This was audio recorded and transcribed. Written consent was obtained from the FGD participants for using their responses.

\section{Analysis}

We gathered results from three sources: a pre-workshop stress scale, responses from the workshop and a post-workshop focus group discussion. Scores from the stress scale were calculated and assigned categories of mild, moderate and severe. We used the Fischer exact test to calculate association of stress with gender, specialty and year of training. The scale was also analyzed for items linked to perceived selfefficacy and helplessness (15). A p-value of $<0.05$ was considered statistically significant. We performed 
a documentary analyses of workshop responses and a thematic analysis of the FGD. Each member of the research team read both the transcripts, identified repeated words and phrases for coding and generated themes for analysis. We also compared workshop feedback with the FGD responses to gauge long term perceived effectiveness of the workshop.

\section{Results}

A total of 49 post-graduate trainees participated, from the departments of Family Medicine, Emergency Medicine, Internal Medicine, Radiology, Obstetrics \& Gynecology and Pathology, of which 27 consented to share information; 19 were females. Twenty-four participants completed the stress scale with scores ranging from 4 to $24 ; 56 \%$ participants were in the moderate stress range and the rest in low stress range.

No statistically significant association was found between overall stress level and gender, specialty or year of training. A statistically significant relationship was observed between stress level and perceived helplessness ( $p$ value 0.002). There was an inverse relationship between stress level and self-efficacy, with a $p$ value of 0.053 . The relationship between self-efficacy and perceived helplessness is depicted in Fig. 1, however, this was not found to be statistically significant.

Three themes were identified for each of the 3 components of $A B C$ model which are described in Table 1. 
Table 1

Themes from $A B C$ model

\section{Components of Percentage Examples ABC Model}

\section{Adversity}

$\begin{array}{lll}\text { Social } & 37 \% & \text { Relationship with family \& friends } \\ \text { Professional } & 75 \% & \begin{array}{l}\text { Increased workload, working outside of normal role, disruption in } \\ \text { regular training and disruption of future career plans }\end{array}\end{array}$

Emotional $\quad 37 \% \quad$ Fear, anxiety, a sense of detachment and uncertainty

\section{Beliefs}

$\begin{array}{lll}\text { Personal } & \sim 33 \% & \text { Implications for self \& family } \\ \begin{array}{l}\text { Situation at } \\ \text { large }\end{array} & \sim 50 \% & \text { Negative perception, 'maybe doomsday is near' } \\ & \begin{array}{l}\text { Couple of participants were hopeful, '... it will soon be back to } \\ \text { normal.' }\end{array}\end{array}$

Situation at $\quad 33 \% \quad$ Disruption of function at work
work

\section{Consequences}

\begin{tabular}{lll} 
Feelings & $\sim 50 \%$ & Anxiety, sadness \& anger \\
\hline Relationships & $>50 \%$ & Negative effect on personal \& professional relationships \\
& $25 \%$ & Unaffected or saw this an opportunity to strengthen relationships \\
$\begin{array}{l}\text { Actions \& } \\
\text { behaviors }\end{array}$ & $20 \%$ & $\begin{array}{l}\text { Unhelpful coping behaviors: deliberate self-isolation, stressful } \\
\text { eating, avoiding family \& friends } \\
\text { Helpful coping behaviors: indulging in hobbies, spending time with } \\
\text { family \& friends } \\
\text { Turned to prayers as a coping mechanism }\end{array}$ \\
\hline
\end{tabular}

In the first step to reframing, "accepting the uncontrollable" theme focused on loss of control of lives, rapid disease spread, social distancing and work disruption. Themes from step 2 "focusing on the controllable" and step 3, "acknowledging own strengths", are depicted in Figs. 2 \& 3. As a fourth step in reframing, participants derived some positives from their strengths. About $20 \%$ wanted to improve their self-efficacy as professionals and the rest considered this situation as an opportunity for personal growth including spirituality, reflection, strengthening of relationships and self-development.

The feedback of the workshop was largely positive, some of which were, sharing adverse experiences, developing a different perspective and a newer way of processing feelings. Some negatives included limited time and number of workshops. About $10 \%$ of the participants did not find this workshop useful. 
The themes that emerged from recurring responses in FGD were as follows: The first centered on the stress scale. Most respondents found the scale helpful for stress quantification. The second theme focused on the ABC tool which participants found useful, however a need for repeated practice was reported. Most participants found reframing of thoughts to be easier. The impact was not immediate but helped reduce stress over time. As a participant stated '...in the long term I think my stress got much less because I kept on recalling the positive points that I had identified.'

\section{Discussion}

This study aimed to share experiences of post-graduate trainees with an intervention for mental wellbeing during COVID-19 pandemic. While other support programs for stress reduction in healthcare workers have been implemented, a reluctance in use has been reported(8). In our setting, we chose to recruit trainees for active participation and utilization of an intervention.

Cognitive behavior therapy based intervention has proven efficacy in stress reduction(16). We, for the first time, introduced a group CBT intervention at our institution, which was well-received. This strategy is effective both in stress reduction and self-efficacy(17). Other strategies like empathic listening and mindfulness also have a role in stress reduction but require greater time and commitment from participants $(18,19)$. The $A B C$ model also had the advantage of ease of use for participants who were mostly naive to this approach.

Opening the workshop with sharing the overall stress level helped set the stage for the interaction between participants. Moderate stress as seen in our study has been reported in postgraduate trainees and other healthcare workers $(20,21)$. Residents with higher stress had a higher perceived helplessness \& lower self-efficacy, and vice versa. The relationship between increased rates of burn-out, high stress and low efficacy has been found in post-graduate trainees $(22,23)$ and further highlights the need for resilience building and support programs.

The participants reported that the stress scale equipped them with a tool to quantify their stress, however, stress level varied. As a participant stated, 'quantifying our stress at one place in time was helpful.' Another stated 'My stress level varies at different times.'

The workshops allowed participants to deconstruct their individual stressors. The reasons for stress were largely similar across all six training programs and to those reported in other studies $(24,25)$ suggesting a collective experience for trainees across varying locations.

The experience of "A-adversity" (in the ABC model) being collective in this case, allowed most participants to share personal stories and accounts during the workshop. This exchange of experiences also validated emotions and beliefs. As quoted by two participants: 'the good thing was to listen to others speak about the COVID experience they had', 'That everyone is going through difficult time, not only me.' 
The reframing of stress was found to be very useful as quoted 'The reframing part was a very good strategy to help oneself...' Another participant stated, 'Ilearnt the reframing technique, where you focus on the controllable and your strengths. Had I known this in past, many days of futile overthinking would not have happened.'

Use of a CBT model was however a completely new strategy for the trainees. As one participant said, 'Actually it was the first time that somebody made us actually think about what stress is right now and how we can cope with it'. Another trainee stated, 'So this was a new thing for us.'

For those who were offered a follow-up workshop, the concept and process became easier to use. As quoted 'I think when we practice again and again, we can apply this better.' Other participants also highlighted the need to have more time in a session and to have a series of workshops for better understanding of the concept and its application.

The reflective exercise was particularly helpful and participants recognized its application in future stressful events. In the words of one participant, 'I will try to incorporate reflection into my daily life so I can better understand what is going on and how I can effectively cope with a situation and help others.' Reflection has been suggested as a way to improve learning from a workshop and its impact on practice (26).

At our institution, this was an initial attempt to formally introduce cognitive reframing for stress management and coping. Not much literature exists on use of such techniques in similar programs. Emotional well-being of postgraduate trainees has implications on patient safety, communication skills and professionalism(27); essential competencies of the Accreditation Council for Graduate Medical Education (28). With increasing rates of burn-out in healthcare workers and trainees(29) and emergence of global health crises, inclusion of such techniques as part of self-care and resilience building becomes increasingly important(30).

Study limitations

This study was an attempt to introduce a cognitive tool for coping and resilience. While the individual responses affirmed that self-understanding of stress was helpful, a post-workshop stress was not measured, which would have strengthened the study findings. Additionally, the small sample may have limited our ability to derive concrete results. Triangulation of the three data sets, however, did help validate the usefulness of the workshop in stress alleviation while offering a method for resilience building.

\section{Conclusion}

The current pandemic has caused significant stress in healthcare workers, impacting professional and personal lives. Group CBT proved to be a useful tool in recognizing and managing stress. Such 
interventions can be incorporated in training programs to help reduce stress and build resilience for similar events.

\section{Abbreviations}

PTSD: Post Traumatic Stress Disorder

SARS: Severe Acute Respiratory Syndrome

CBT: Cognitive Behavior Therapy

PSS: Perceived Stress Scale

TECHS: Toolkit for Emotional Coping for Healthcare Staff

FGD: Focus Group Discussion

\section{Declarations}

\section{Ethics approval and consent to participate:}

The proposal was submitted to the Ethics Review Committee of the Aga Khan University, Karachi, Pakistan, and approval was obtained. All methods were performed in accordance with the relevant guidelines and regulations. We included the data of the participants who provided written informed consent for this study.

\section{Consent for publication:}

Not applicable

\section{Availability of data and materials:}

The datasets used and analyzed during the current study are available from the corresponding author on reasonable request.

\section{Competing interests:}

The authors declare that they have no competing interests.

\section{Funding:}




\section{Authors' contributions:}

SS conceived the study and wrote the manuscript. RI performed the data analysis and co-wrote the manuscript. Both authors read and approved the final manuscript.

\section{Acknowledgements:}

We acknowledge contribution of Dr Imran Hassan, Instructor, Department of Family Medicine, Aga Khan University, for his help with data analysis.

\section{References}

1. Chew NW, Lee GK, Tan BY, Jing M, Goh Y, Ngiam NJ, et al. A multinational, multicentre study on the psychological outcomes and associated physical symptoms amongst healthcare workers during COVID-19 outbreak. Brain, behavior, and immunity. 2020;88:559-65.

2. Luo Y, Wu J, Lu J, Xu X, Long W, Yan G, et al. Investigation of COVID-19-related symptoms based on factor analysis. Annals of Palliative Medicine. 2020.

3. Xiong J, Lipsitz O, Nasri F, Lui LM, Gill H, Phan L, et al. Impact of COVID-19 pandemic on mental health in the general population: A systematic review. Journal of affective disorders. 2020.

4. Naushad VA, Bierens JJ, Nishan KP, Firjeeth CP, Mohammad OH, Maliyakkal AM, et al. A systematic review of the impact of disaster on the mental health of medical responders. Prehospital and disaster medicine. 2019;34(6):632-43.

5. Tam CW, Pang EP, Lam LC, Chiu HF. Severe acute respiratory syndrome (SARS) in Hong Kong in 2003: stress and psychological impact among frontline healthcare workers. Psychological medicine. 2004;34(7):1197.

6. da Silva FCT, Neto MLR. Psychological effects caused by the COVID-19 pandemic in health professionals: a systematic review with meta-analysis. Progress in Neuro-Psychopharmacology and Biological Psychiatry. 2020:110062.

7. Chirico F, Nucera G, Magnavita N. Protecting the mental health of healthcare workers during the COVID-19 emergency. BJPsych International. 2020:1-2.

8. Chen Q, Liang M, Li Y, Guo J, Fei D, Wang L, et al. Mental health care for medical staff in China during the COVID-19 outbreak. The Lancet Psychiatry. 2020;7(4):e15-e6.

9. Liu S, Yang L, Zhang C, Xiang Y-T, Liu Z, Hu S, et al. Online mental health services in China during the COVID-19 outbreak. The Lancet Psychiatry. 2020;7(4):e17-e8.

10. David OA, Matu SA, Pintea S, Cotet CD, Nagy D. Cognitive-behavioral processes based on using the ABC analysis by trainees' for their personal development. Journal of Rational-Emotive \& Cognitive- 
Behavior Therapy. 2014;32(3):198-215.

11. Kar N. Cognitive behavioral therapy for the treatment of post-traumatic stress disorder: a review. Neuropsychiatric disease and treatment. 2011.

12. Barrera T, Mott J, Hofstein R, Teng E. A meta-analytic review of exposure in group cognitive behavioral therapy for posttraumatic stress disorder. Clinical psychology review. 2013;33(1):24-32.

13. Cohen S, Kamarck T, Mermelstein R. Perceived stress scale. Measuring stress: A guide for health and social scientists. 1994;10:1-2.

14. Price J K-AN, Kazak A,. Toolkit for Emotional Coping for Healthcare Staff (TECHS):Helping Healthcare Workers Cope with the Demands of COVID-19 2020 [cited 2020 December 2020]. Available from: https://djph.org/wp-content/uploads/2020/08/4_Price_TECHS.pdf.

15. Roberti JW, Harrington LN, Storch EA. Further psychometric support for the 10-item version of the perceived stress scale. Journal of College Counseling. 2006;9(2):135-47.

16. Ruotsalainen J, Serra C, Marine A, Verbeek J. Systematic review of interventions for reducing occupational stress in health care workers. Scandinavian journal of work, environment \& health. 2008:169-78.

17. Jafar HM, Salabifard S, Mousavi SM, Sobhani Z. The effectiveness of group training of cbt-based stress management on anxiety, psychological hardiness and general self-efficacy among university students. Global journal of health science. 2016;8(6):47.

18. Rodriguez-Vega B, Palao Á, Muñoz-Sanjose A, Torrijos M, Aguirre P, Fernández A, et al. Implementation of a Mindfulness-based crisis intervention for frontline healthcare workers during the COVID-19 outbreak in a public general hospital in Madrid, Spain. Frontiers in psychiatry. 2020;11:1170.

19. Gaspar I, Martinho A, Lima M. Exploring the benefits of a mindfulness program for Portuguese public healthcare workers. Current Psychology. 2018:1-10.

20. Imran N, Masood HMU, Ayub M, Gondal KM. Psychological impact of COVID-19 pandemic on postgraduate trainees: a cross-sectional survey. Postgraduate medical journal. 2020.

21. Wang Y, Li Y, Jiang J, Feng Y, Lu D, Zhang W, et al. COVID-19 outbreak-related psychological distress among healthcare trainees: a cross-sectional study in China. BMJ open. 2020;10(10):e041671.

22. Janko MR, Smeds MR. Burnout, depression, perceived stress, and self-efficacy in vascular surgery trainees. Journal of vascular surgery. 2019;69(4):1233-42.

23. Smeds MR, Janko MR, Allen S, Amankwah K, Arnell T, Ansari P, et al. Burnout and its relationship with perceived stress, self-efficacy, depression, social support, and programmatic factors in general surgery residents. The American Journal of Surgery. 2020;219(6):907-12.

24. Collins C, Mahuron K, Bongiovanni T, Lancaster E, Sosa JA, Wick E. Stress and the Surgical Resident in the COVID-19 Pandemic. Journal of surgical education. 2020.

25. Sneyd JR, Mathoulin SE, O'Sullivan EP, So VC, Roberts FR, Paul AA, et al. The impact of the COVID-19 pandemic on anaesthesia trainees and their training. British journal of anaesthesia. 2020. 
26. Moon J. Using reflective learning to improve the impact of short courses and workshops. Journal of continuing education in the health professions. 2004;24(1):4-11.

27. Ripp JA, Privitera MR, West CP, Leiter R, Logio L, Shapiro J, et al. Well-being in graduate medical education: a call for action. Academic Medicine. 2017;92(7):914-7.

28. Arora S, Ashrafian H, Davis R, Athanasiou T, Darzi A, Sevdalis N. Emotional intelligence in medicine: a systematic review through the context of the ACGME competencies. Medical education. 2010;44(8):749-64.

29. Robinson DBT, James OP, Hopkins L, Brown C, Powell A, Abdelrahman T, et al. Trainee burnout: when does the fire start? Postgraduate Medical Journal. 2020.

30. Wald HS. Optimizing resilience and wellbeing for healthcare professions trainees and healthcare professionals during public health crises-Practical tips for an 'integrative resilience'approach. Medical Teacher. 2020;42(7):744-55.

\section{Figures}




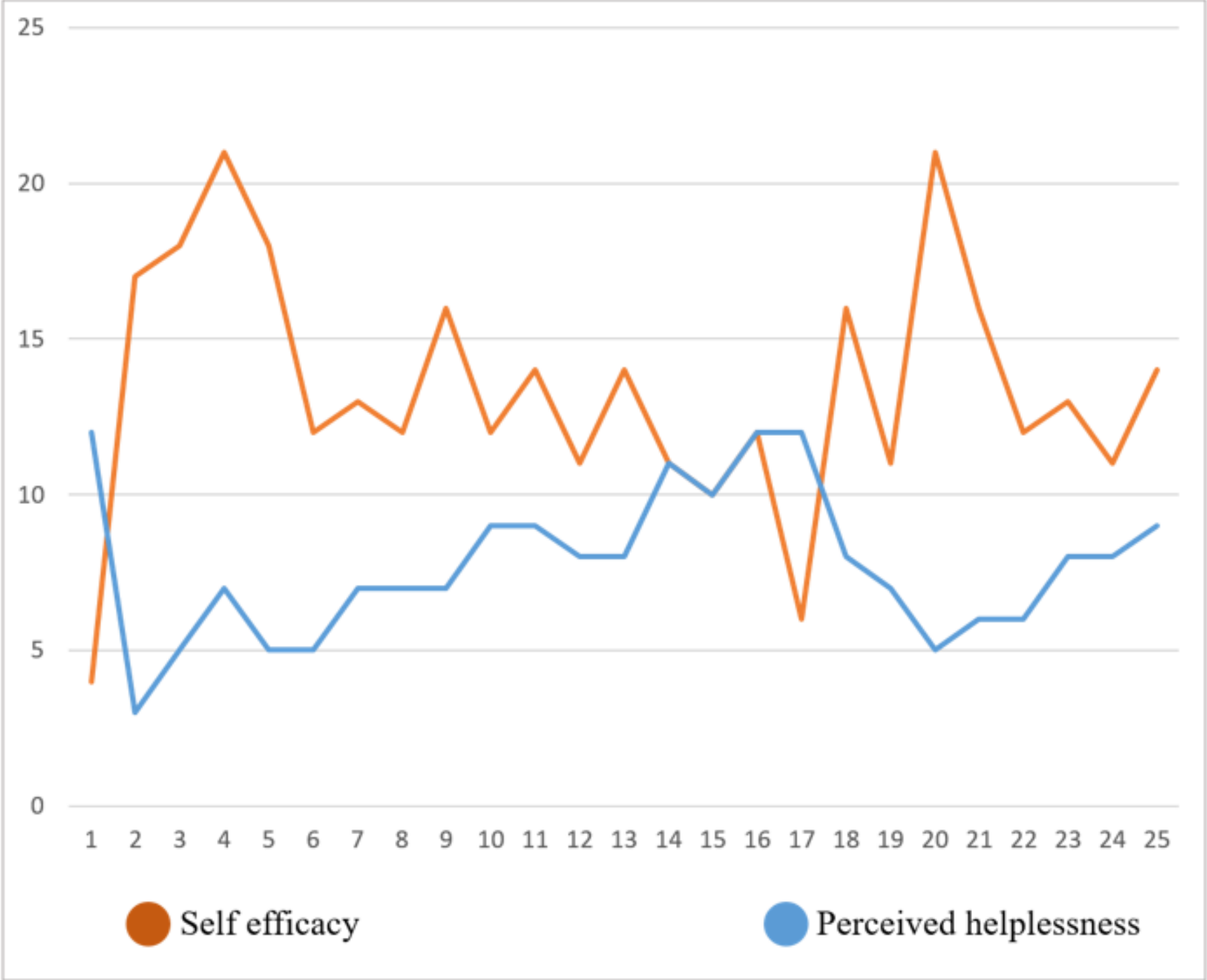

Figure 1

Stress Scale Analysis 
Within my control

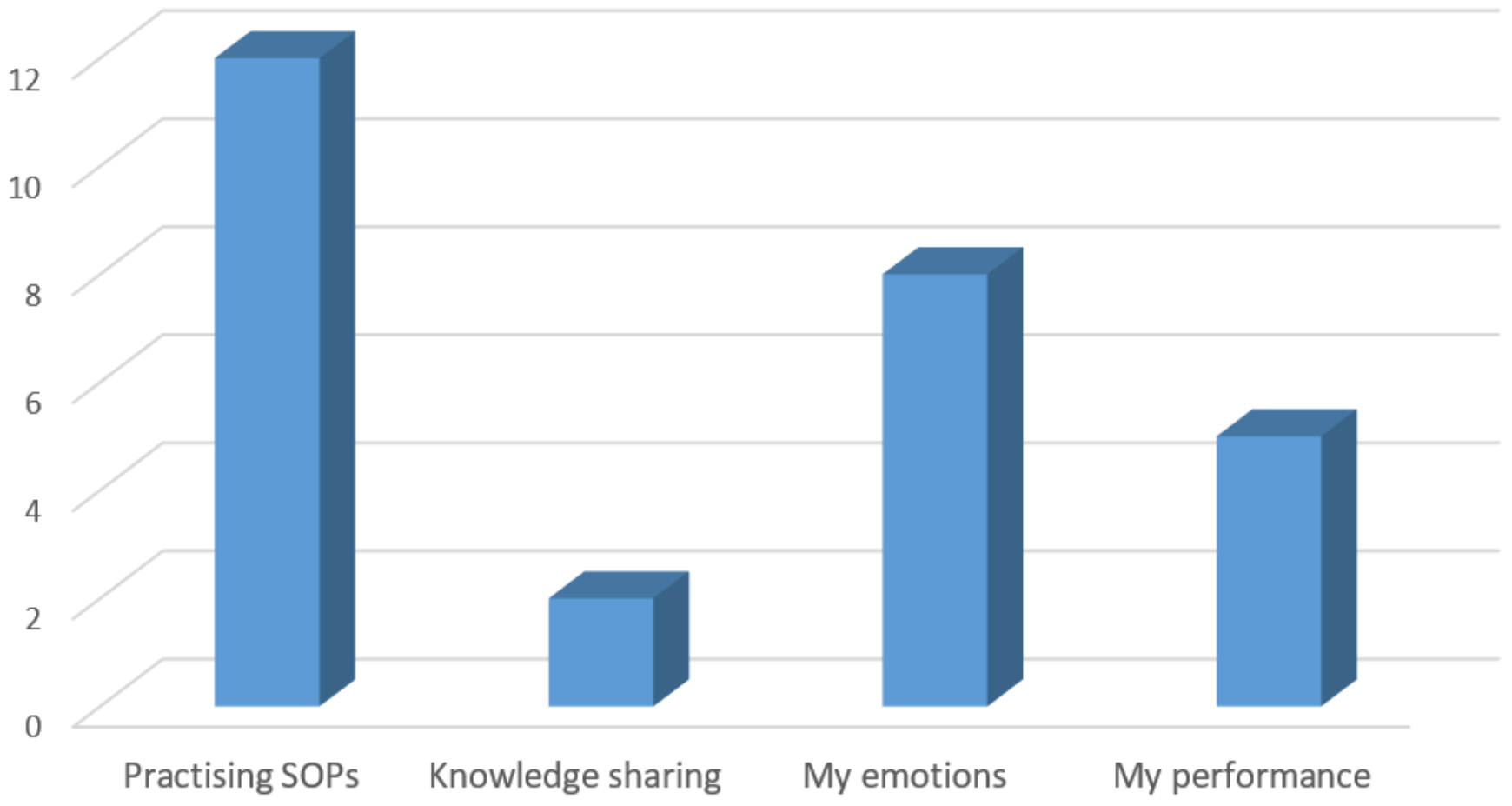

Figure 2

Step 2 of Reframing 'Focusing on the Controllable'

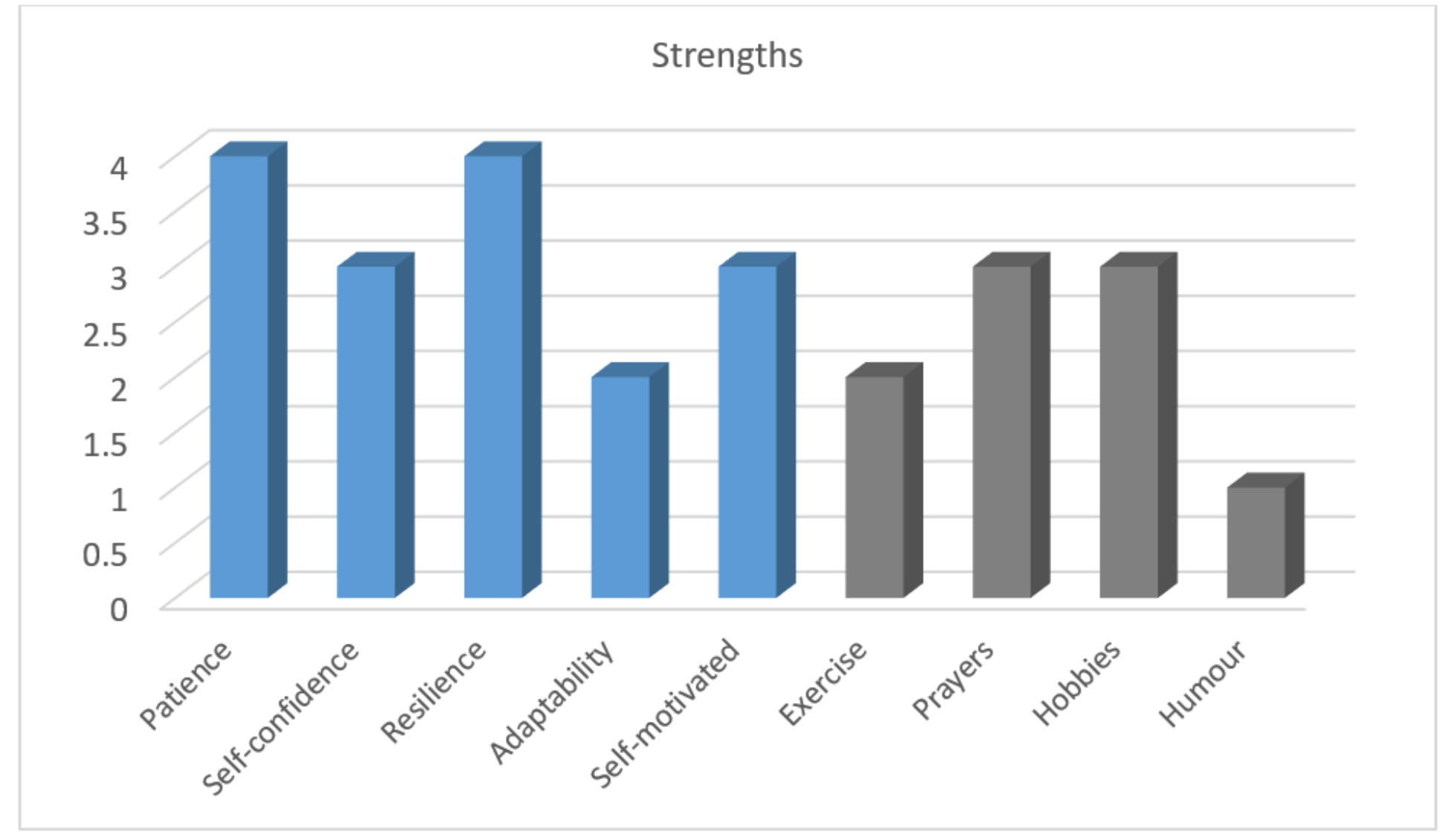


Figure 3

Step 3 of Reframing 'Acknowledging own Strengths' 